\title{
Der Nutzer im Rad der Zeit
}

\section{Die Durchführung einer Tagebuchstudie am Fallbeispiel}

\author{
Patricia Paule \\ Universität Regensburg \\ Universitätsstraße 31 \\ 93053 Regensburg \\ PatriciaPa@outlook.de
}

Tim Schneidermeier

Universität Regensburg

Universitätsstraße 31

93053 Regensburg

tim.schneidermeier@gmail.com

\author{
Sabrin Kenaan \\ Universität Regensburg \\ Universitätsstraße 31 \\ 93053 Regensburg \\ sabrin.kenaan@gmail.com
}

\begin{abstract}
Die Methodik der Diary Study bietet die Möglichkeit, über einen definierten längeren Zeitraum hinweg den zu untersuchenden Gegenstand in der natürlichen Umgebung des Nutzers zu evaluieren. Diese Herangehensweise bringt gegenüber herkömmlichen Evaluationsmethoden den Vorteil mit sich, die User Experience (UX) der Teilnehmer unbeeinflusst von den Einschränkungen einer zeitlich begrenzten Testsituation einfangen zu können. Anhand einer Fallstudie zur Langzeitevaluation mobiler Anwendungen werden Erkenntnisse und Lessons Learned bei der Gestaltung und konkreten Umsetzung einer Tagebuchstudie erörtert.
\end{abstract}

\section{Keywords}

Diary Study, Langzeitstudie, Evaluationsmethodik, Usability, User Experience, Lessons Learned

\section{Einleitung}

Es existieren zahlreiche Möglichkeiten, die Usability eines Produkts zu evaluieren. Der Nutzungskontext des zu untersuchenden Gegenstandes stellt hierbei einen maßgeblichen Einflussfaktor auf die Entscheidung für die jeweils passende Evaluationsform dar. Im Falle des vorliegenden Projektes handelt es sich bei den Evaluationsgegenständen um die mobile Nachrichten- und ePaper-App einer Regensburger Zeitung. Trotz hoher Kosten und des hohen Auswertungsaufwandes, die das Durchführen einer Langzeitevaluation mit sich bringt, 
wurde sich bewusst für die Untersuchung in Form einer Diary Study entschieden. Im Gegensatz zu Momentaufnahmen traditioneller Testverfahren ermöglicht dieses Format, den Umgang der Probanden mit den Applikationen im Kontext ihres gewohnten Tagesablaufs ohne die direkte und indirekte Einflussnahme von Testleiter und Laborsituation auf die Testergebnisse und das Nutzungserlebnis aufzuzeichnen bzw. aufzeichnen zu lassen. Somit entsteht ein natürlicher und spontaner Eindruck der User Experience. Ebenso kann eine mögliche Veränderung der UX von der Erstnutzung bis zum Studienende festgehalten werden. Durch das Führen eines (UX-)Tagebuchs wird eine große Menge qualitativer und quantitativer Daten erhoben. Diese erfassen sowohl Eindrücke zum aktuellen Nutzungszeitpunkt als auch die Entwicklung über den gesamten Evaluationszeitraum hinweg sowie mögliche zu dieser Entwicklung korrelierenden Gründe. Werkzeuge und Plattformen für eine Diary Study gibt es viele: Von der analogen Version mit Stift und Zettel, über Email-Austausch, Blogging und Twitter bis hin zu einer Mischform oder dem Einsatz kostenpflichtiger professioneller Tools (Santafe, 2011). Das Format der Tagebuchstudie verlangt umfassende Vorüberlegungen und eine zielgerichtete Planung der Studie, denn die Organisatoren riskieren sonst schnell einen zu hohen Auswertungsaufwand (Lallemand, 2012). Auch der Auswahl der Probanden sollte Aufmerksamkeit zukommen, da sie ein großer Arbeitsaufwand erwartet und ein entsprechend hoher Grad an Zuverlässigkeit gewährleistet sein sollte (Sapounakis, 2011). Der Fokus der folgenden Ausführungen liegt auf der Beschreibung von Vor-und Herangehensweisen an die Durchführung einer solchen Tagebuchstudie und leitet aus den Ergebnissen resultierende Erfahrungen bzw. Lessons Learned ab.

\section{Fallstudie: Langzeitevaluation einer mobilen Nachrichten- und ePaper-App}

Ziel der betrachteten Diary Study war herauszufinden, ob die UX bei der Nutzung der Apps der der Nutzung einer Papierzeitung entspricht bzw. das Papierformat sogar ablösen könnte. Die zehntägige Studie, die im Rahmen eines universitären Projektes stattfand, umfasste acht Studienteilnehmer. Einleitung und Abschluss der Diary Study bildeten Vor-und Nachinterviews der Teilnehmer. Ein Usability-Test zu Studienbeginn diente als Grundlage für einen Vergleich zwischen Erstnutzungs-Erfahrung und den möglichen Veränderungen der Interaktions-Erfahrungen (Lernkurve) über den Evaluationszeitraum hinweg. Bereits in einem frühen Stadium der Studienplanung wurde aufgrund der folgenden positiven Aspekte festgelegt, die Tagebuchstudie in Form eines Blogs durchzuführen: Die Möglichkeit der Live-Überwachung, die dynamische Anpassbarkeit und das digitale Datenformat. Um einen möglichst umfassenden Eindruck der UX der Probanden gewinnen, sollten die Tagebucheinträge im vorliegenden Fall in Freitext-Form formuliert werden. Um dennoch alle für die Studie essentiellen Informationen einholen zu können, wurden den Testern strukturelle und inhaltliche Vorgaben sowie Beispieleinträge an die Hand gegeben. Um dem gänzlichen Ausfall von Tagebucheinträgen vorzubeugen und die Motivation der Studienteilnehmer zu erhalten, wurde der Blog durch Informationen zur Bedienung, zum Verlauf der Studie und um die Kontaktdaten der Organisatoren ergänzt. Fun Facts dienten 
zur Unterhaltung der Probanden und wurden mit den bearbeitbaren Beitragsvorlagen jeden Tag für die Probanden freigeschalten. Die Dokumente wurden in einen für jeden Probanden erstellten Zeitplan eingebunden, der zur allgemeinen Orientierung, Erinnerung und durch die abhakbaren Module auch zur Motivation der Teilnehmer beitragen sollte. Zwei Tage, an denen keine Fragebögen zu bearbeiten waren, enthielten jeweils zwei konkrete Tasks, die die Navigationsstruktur und Informationsarchitektur der Anwendungen untersuchen und potentielle Veränderungen der UX bzw. Usability aufdecken sollten. Zwei Wochen nach Abschluss der Testphase wurden die Probanden außerdem darum gebeten, in einem abschließenden Fragebogen ihren Gesamteindruck von der Studie selbst festzuhalten. Es fand ein stetiger Mailverkehr seitens der Organisatoren statt, um die Teilnehmer zu informieren, zu motivieren und gegebenenfalls an das Verfassen der Einträge zu erinnern.

\section{Lessons Learned}

\section{Positive Erkenntnisse}

- Die Plattform des Blogs für die Durchführung der Studie wurde von den Probanden zu $100 \%$ befürwortet und die Dauer von 10 Tagen wurde als angemessen empfunden.

- Die vorgefertigten Bloginhalte und der eingebundene Stundenplan wurden als (sehr) hilfreich bewertet. Die Vorlagen (Struktur/Aufbau, Einsatz der Smileys/Substantive) wurden für die eigenen Einträge berücksichtigt und größtenteils als zur Übernahme verpflichtend verstanden.

- Die Teilnehmer sprachen sich den Fun Facts ${ }^{27}$ gegenüber positiv aus und auch die gestellten Tasks fanden zu 100\% Anklang.

- Die Rückmeldung per Mail von Seiten der Organisatoren wurden als „sympathisch“ und ,motivierend“ beschrieben und alle Befragten würden noch einmal an einer Diary Study teilnehmen.

- Mit Hilfe des Eingangs durchgeführten Usability-Tests konnte insgesamt eine positive Entwicklung der UX der Probanden im Umgang mit den Apps festgestellt werden.

- Der Einsatz eines AttrakDiff mit A/B-Vergleich bot auch bei geringer Teilnehmerzahl die Möglichkeit, allgemeine Tendenzen zu visualisieren, die im weiteren Kontext der Arbeit hilfreich waren.

\section{Ergänzende Handlungsempfehlungen}

Im Weiteren werden die aus verbesserungsfähigen Aspekten der Studie abgeleiteten Handlungsempfehlungen aufgeführt:

27 Die Einbindung unterhaltsamer Fakten zu historischen Ereignissen am jeweiligen Testtag. 
- Ein Usability-Pretest der gewählten Plattform für die Studie gewährleistet einwandfreie Bedienbarkeit und eine positive UX der Probanden schon vor Beginn der Studie.

- Strukturelle und inhaltliche Beispielangaben sollten, falls als solche intendiert, als Richtlinien formuliert und kommuniziert werden.

- Bei geringer Teilnehmerzahl empfiehlt sich bei Fragebögen der Einsatz von Freitextfeldern und Auswahlfragen mit maximal zwei bis drei Optionen anstelle der Verwendung von Skalen und Mehrfachauswahlen, um eindeutigere Resultate zu erzielen.

- Das zeitintensive und aufwendige Verfassen von Freitext-Blogeinträgen könnte auf jeden zweiten Studientag reduziert und dennoch als hilfreiches Werkzeug zur Evaluation der UX eingesetzt werden, wenn

- $\quad$ für die Auswertung grundlegende Informationen direkt über vorgegebene Fragen bzw. zielgerichtet konzipierte Auswahlskalen (Emoticons, Bewertung von Funktionen und Designentscheidungen etc.) eingeholt werden.

- Die Dauer einer Langzeitstudie sollte an den Funktionsumfang der zu testenden Anwendungen angepasst werden.

- Anstelle der ausschließlichen Anleitung der Probanden via Mail, könnte ein Kick-OffTreffen mit den Teilnehmern, in dem gemeinsam die Rahmenbedingungen, Ziele und Vorgaben, die eingesetzte Hard- und Software sowie der konkrete Projektablauf besprochen und offene Fragen geklärt werden einen deutlichen Mehrwert für die Ergebnisse der Studie und Zufriedenheit der Teilnehmer generieren.

- Weitere Maßnahmen zur Verbesserung der Studienbilanz wären die Optimierung des Blogs für mobile Endgeräte und das Einbinden multimedialer Inhalte als konkreter Studienbestandteil über Apps mit Snippet-Funktionen, Audio- und Videoaufnahmen.

\section{Fazit}

Die Durchführung einer Diary Study bedeutet einen hohen Planungs-und Organisationsaufwand und eine Zieldefinition zu Beginn der Studie ist unerlässlich. Sorgfältig und mit entsprechendem Aufwand durchgeführt hilft der Ansatz vor allem, explizit herauszufiltern, aus welchen Gründen Applikationen zu welchem Zeitpunkt genutzt oder nicht genutzt werden und gibt zudem über den jeweiligen Gefühlszustand der Anwender detailliert Auskunft. Auf diese Weise können persönliche Emotionen eingefangen werden, die bei einer herkömmlichen Usability-Testsituation im Labor nicht zu erfassen sind. Im Rahmen einer Diary Study lassen sich nicht nur allgemeine Usability-Mängel feststellen, die Einfluss auf die User Experience bei der Erstnutzung nehmen: Es werden Erwartungen an und daraus resultierende Verbesserungsmöglichkeiten für die Applikationen über den Studienzeitraum evaluiert, deren Umsetzung und Einarbeitung die UX der (zukünftigen) Nutzer vor allem hinsichtlich langfristiger Nutzung befriedigen wird. 


\section{Literatur}

Lallemand, C. (2012). Dear Diary: Using Diaries to Study User Experience. URL: http://uxpamagazine.org/dear-diary-using-diaries-to-study-user-experience/

Santafe, I. (2011). Diary Study guide: how to get the best results from diary study research. URL: http://www.webcredible.com/blog-reports/web-usability/diary-study-guide.shtml

Sapounakis, E. (2011). The Do's and Don'ts of Diary Studies. URL:

http://www.eriontheinterweb.com/2011/07/the-dos-and-donts-of-diary-studies/ 